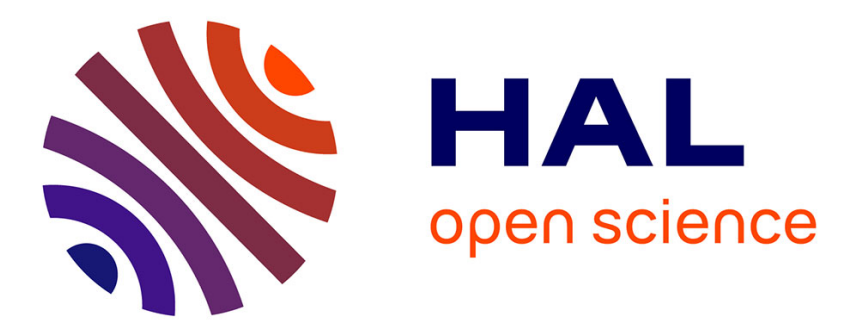

\title{
Periodic modulations controlling Kuznetsov-Ma soliton formation in nonlinear Schrödinger equations
}

Gaston G L Tiofack, Saliya Coulibaly, Majid Taki, Stephan de Bièvre, Guillaume Dujardin

\section{> To cite this version:}

Gaston G L Tiofack, Saliya Coulibaly, Majid Taki, Stephan de Bièvre, Guillaume Dujardin. Periodic modulations controlling Kuznetsov-Ma soliton formation in nonlinear Schrödinger equations. Physics Letters A, 2017, 10.1016/j.physleta.2017.04.029 . hal-01403028

\section{HAL Id: hal-01403028 \\ https://hal.inria.fr/hal-01403028}

Submitted on 25 Nov 2016

HAL is a multi-disciplinary open access archive for the deposit and dissemination of scientific research documents, whether they are published or not. The documents may come from teaching and research institutions in France or abroad, or from public or private research centers.
L'archive ouverte pluridisciplinaire HAL, est destinée au dépôt et à la diffusion de documents scientifiques de niveau recherche, publiés ou non, émanant des établissements d'enseignement et de recherche français ou étrangers, des laboratoires publics ou privés. 


\title{
Periodic modulations controlling Kuznetsov-Ma soliton formation in nonlinear Schrödinger equations
}

\author{
C. G. L. Tiofack, S. Coulibaly, and M. Taki \\ Univ. Lille, CNRS, UMR 8523 - PhLAM - Physique des Lasers Atomes et Molécules, F- 59000 Lille, France \\ S. De Bièvre and G. Dujardin \\ Univ. Lille, CNRS, UMR 8524 - Laboratoire Paul Painlevé, F-59000 Lille, \\ France; Équipe-Projet Mephysto, INRIA Lille-Nord Europe, France
}

\begin{abstract}
We analyze the exact Kuznetsov-Ma soliton solution of the one-dimensional nonlinear Schrödinger equation in the presence of periodic modulations satisfying an integrability condition. We show that, in contrast to the case without modulation, the Kuznetsov-Ma soliton develops multiple compression points whose number, shape and position are controlled both by the intensity of the modulation and by its frequency. In addition, when this modulation frequency is a rational multiple of the natural frequency of the Kutzetsov-Ma soliton, a scenario similar to a nonlinear resonance is obtained: in this case the spatial oscillations of the Kuznetsov-Ma soliton's intensity are periodic. When the ratio of the two frequencies is irrational, the soliton's intensity is a quasiperiodic function. A striking and important result of our analysis is the possibility to suppress any component of the output spectrum of the Kuznetsov-Ma soliton by a judicious choice of the amplitude and frequency of the modulation.
\end{abstract}

\section{INTRODUCTION}

During the last decades, rogue waves (also known as freak waves), which originally designated abnormally high sea surface waves, have been shown to arise, and consequently gained increasing interest, in various physical systems [1]. In addition to being found in the deep ocean, rogue waves have thus been discovered in optical systems [2, 3], capillary flow [4], Bose-Einstein condensates [5], plasmas [6] and the atmosphere [7]. Rogue waves, which have a peak amplitude generally more than twice the average wave height, appear from nowhere and disappear without a trace [8]. Although their fundamental origin has not been understood completely, there is consensus [9] that rogue waves can be intimately associated with certain types of breathers of the underlying evolution equations. Breathers have drawn a lot of attention in this context because of their interaction and energy exchange with a constant background [10-12]. Such interactions produce unique behaviour that is different from bright soliton dynamics.

In many circumstances, the nonlinear waves under study can be accurately described by solutions of integrable one-dimensional nonlinear Schrödinger equations (NLSE) with constant coefficients and their exact analytical breather solutions have been interpreted as rogue waves [13]. Breather solutions on a finite background that have been identified are the Kuznetsov-Ma (KM) soliton [14, 15], which is localized in the transverse direction, the Akhmediev breather $(\mathrm{AB})$ [16], localized in the evolution direction, and the Peregrine soluton [17], which is a limiting case of the KM soliton and the $\mathrm{AB}$, localized in both the transverse and evolution directions. Extensive theoretical and experimental studies have been devoted to characterize and control the formation of these solutions $[18,19]$. This includes collisions of ABs [20], the optimum conditions for the experimental generation of the Peregrine soliton [2] and of the KM soliton [21].

Rogue waves and breathers in variable coefficient nonlinear partial differential equations have attracted considerable interest recently and have been shown to present a rich variety of features. For example, recent studies have reported breather evolution, amplification and compression, Talbot-like effects and composite rogue wave structures in the variable coefficient derivative NLSE [22]. Experimental study has furthermore shown that the properties of the $\mathrm{AB}$ can be affected by a varying dispersion [23]. The appearance of a novel multiple compression point structure in a periodically modulated NLSE, referred to as a Peregrine comb, has been reported recently [24]. The controllable behavior, including partial excitation, maintenance, and postponement of the Peregrine, the $\mathrm{AB}$ and $\mathrm{KM}$ solitons have also been discussed in certain variable coefficient NLSE $[25,26]$ describing dispersion decreasing fibers. Additionally, the modulational instability of the $\mathrm{KM}$ soliton in optical fibers with periodic dispersion was investigated in [27].

In this paper, we shall study the shape of the KM soliton in a one-dimensional completely integrable NLSE with periodically varying coefficients. We will show that, during the propagation, the spatial dependence or profile of the KM soliton interferes with that of the dispersion modulation and can be controlled by its intensity and period. We show in particular that the KM soliton with periodic coefficients develops multiple compression points whose number and height depend on the strength of the modulation. Further, we take advantage of nonlinear dynamics tools such as phase space analysis and Fourier spectrum to show that, when the frequency of the modulation and the natural frequency of the KM soliton are commensurate, the oscillations of the KM soliton's intensity are periodic. In the case where the two frequecies are incommensurate, the oscillations are quasiperiodic.

The paper is organized as follows. In Sec. II we show 
that under a suitable integrability condition the periodic modulation of the coefficients of the NLSE can lead to a generalized $\mathrm{AB}$, or KM solution with periodic coefficients. In Sec. III the characteristics of the KM soliton with periodic coefficients are investigated. Using Fourier spectrum and stroboscopic Poincaré sections, the different regimes of the oscillations of the KM soliton with periodic coefficients are studied in Sec. IV. Section V contains our concluding remarks.

\section{INTEGRABLE MODEL AND GENERALIZED SOLUTION WITH FINITE BACKGROUND}

We consider the NLSE in the form

$$
i \frac{\partial q}{\partial z}-\frac{D_{2}(z)}{2} \frac{\partial^{2} q}{\partial t^{2}}+R(z)|q|^{2} q=0
$$

where $D_{2}(z)$ and $R(z)$ are both taken to be periodic functions of their argument. In optical fiber systems the above equation describes the dynamics of the envelope $q(z, t)$ of the electric field $[28,29]$ where $z$ and $t$ are the dimensionless propagation distance along the fiber (measured in units of nonlinear length) and dimensionless time, respectively. The function $D_{2}(z)$ represents the GVD coefficient, and $R(z)$ is the nonlinear one. In its general form, Eq. (1) is not integrable. However, if the condition $R D_{2 z}-D_{2} R_{z}=0$ is satisfied, Eq. (1) becomes the integrable NLSE with variable coefficients [30, 31]. Here, we consider the focusing case where $D_{2} R \leq 0$ and assume consequently $D_{2}(z)=-R(z)$. In what follows we will study a soliton solution with finite background of Eq. (1) when the periodic dispersion is of the form

$$
D_{2}(z)=-1+d_{m} \cos \left(k_{m} z\right)
$$

where $d_{m}$ is the amplitude of modulation and $k_{m}$ its spatial frequency. Note that we are not assuming that the perturbations to the dispersion parameter $D_{2}(z)$, and nonlinearity $R(z)$ are small.

In this case, such a solution of (1) can be written as

$$
q(z, t)=\left[1+\frac{2(1-2 a) \cosh (B Z)+i B \sinh (B Z)}{\sqrt{2 a} \cos (\Omega t)-\cosh (B Z)}\right] \exp (-i Z), \quad \begin{aligned}
& \text { III. } \\
& \text { CHARACTERISTICS OF KUZNETSOV-MA } \\
& \text { SOLITON IN PRESENCE OF PERIODIC } \\
& \text { COEFFICIENTS }
\end{aligned}
$$

\section{CHARACTERISTICS OF KUZNETSOV-MA SOLITON IN PRESENCE OF PERIODIC COEFFICIENTS}$$
B=\sqrt{8 a(1-2 a)}, \quad \Omega=2 \sqrt{1-2 a},
$$$$
Z(z)=\int_{z_{\star}}^{z} D_{2}(z) d z
$$

where $z_{\star}$ fixes a global phase and $a$ is a real constant parameterizing this class of the solutions [21]. Without loss of generality, we set $z_{\star}=0$. When $d_{m}=0$, Eq. (3) is reduced to the standard form of the soliton with finite background [16, 32]. For $0<a<1 / 2$, the solutions describe the $\mathrm{AB}$ solutions which exhibit periodic modulations in $t$ with period $2 \pi / \Omega$ and a single growth-decay cycle along $z$. In the limit $a \rightarrow(1 / 2)^{-}$, the solution (3) reduces to the rational Peregrine soliton solution [2]. For $a>1 / 2$, the parameters $B$ and $\Omega$ become imaginary and we set

$$
b=-i B=\sqrt{8 a(2 a-1)} \text { and } \quad \omega=-i \Omega=2 \sqrt{2 a-1} .
$$

The physical nature of the solution (3) changes as circular trigonometric functions become hyperbolic and vice versa. In this case, Eq. (3) reduces to the KM soliton,

$q_{\mathrm{Ma}}(z, t)=\left[1+\frac{2(1-2 a) \cos (b Z)-i b \sin (b Z)}{\sqrt{2 a} \cosh (\omega t)-\cos (b Z)}\right] \exp (-i Z)$,

with

$$
Z=-z+\frac{d_{m}}{k_{m}} \sin \left(k_{m} z\right)
$$

In the case of constant coefficients, Eq. (5) shows localisation in time $t$ but (quasi)-periodicity along $z[14$, 21]. If $b=p / q$ with $p, q$ mutually prime integers, then the solution (5) is periodic with period $2 \pi q=p \times(2 \pi / b)$. Otherwise, it is quasiperiodic. Note that the modulus of $q_{\mathrm{Ma}}$ is always periodic with period $2 \pi / b$ as seen in Figure 1(a). By taking into account the effect of the modulation, the solution (5) is still localized in time but the spatial (quasi)-periodicity is affected by the modulation. For the particular case where the frequency of the modulation $k_{m}$ is a rational multiple of the frequency $b$ of the oscillations in $\left|q_{\mathrm{Ma}}\right|^{2}$, the modulus of the KM solution remains periodic in space (see Fig. 1(b)), otherwise it loses its periodicity (see Fig. 1(c)). In the case with constant coefficients, the periodicity characteristics of the $\mathrm{AB}$ and $\mathrm{KM}$ solutions are different, switching time and space (compare Eq. (3) with $0<a<1 / 2$ and hence real $B$ and $\Omega$ with Eq. (5) with $1 / 2<a$ and hence real $b$ and $\omega)$. As a result, the spatial modulation of the parameters leads to completely different physical mechanisms, as we will see below. In what follows, we will study the KM solution (5) in detail.

We now consider the generalized KM soliton Eq. (5), with $a>1 / 2$, and the periodic dispersion coefficient given by Eq. (2). At a fixed point $z$ one finds that the KM soliton's intensity has a maximum at $t=0$, which is given by

$$
\left|q_{\mathrm{Ma}}\right|_{\max }^{2}(z)=1+\frac{4 \sqrt{2 a}(2 a-1)}{\sqrt{2 a}-\cos (b Z)}
$$

where $Z$ is a function of $z$ defined in (6). As stated previously and observed in Fig. 2, when $d_{m}=0$ the modulus of the KM soliton is periodic in $z$. In the presence of 

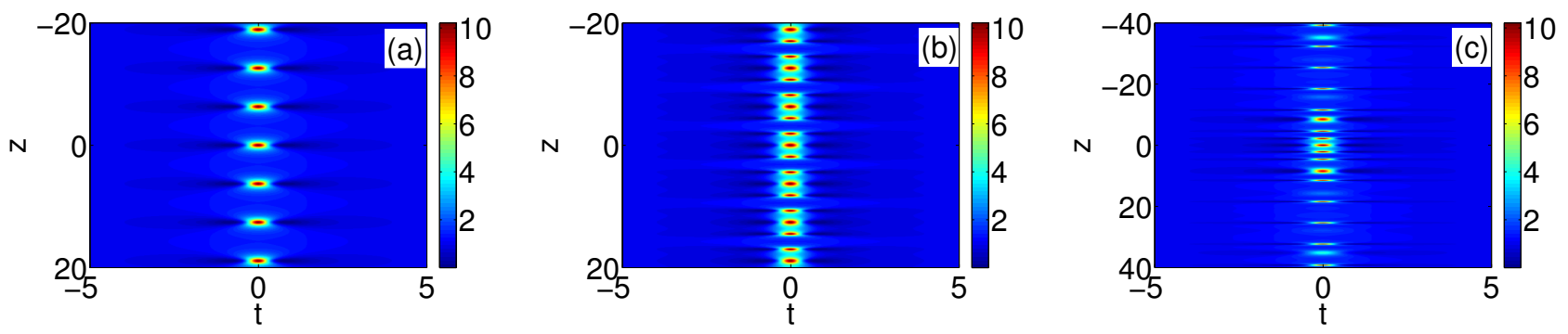

FIG. 1. (Color online) Spatiotemporal evolution of the generalized Kuznetsov-Ma soliton. (a) $d_{m}=0,(\mathrm{~b}) d_{m}=2, k_{m}=b,(\mathrm{c})$ $d_{m}=2, k_{m}=\sqrt{3} b / 2$. Here, $a=0.603$.

modulation $\left(d_{m}>0\right)$, the function $\left|q_{\mathrm{Ma}}\right|_{\max }^{2}$ defined in (7) is periodic provided $k_{m}$ is a rational multiple of $b$. If we set $k_{m} / b=N_{1} / N_{2}$ with $N_{1}$ and $N_{2}$ mutually prime integers, then the period is $z_{*}=2 \pi N_{1} / k_{m}=2 \pi N_{2} / b$. On the other hand, when $k_{m} / b$ is irrational, the function $\left|q_{\mathrm{Ma}}\right|_{\max }^{2}$ is quasiperiodic.

From the above expression we can see that the KM soliton oscillates in space ( $z$-variable) and the maximum intensity value is given by

$$
P=1+\frac{4 \sqrt{2 a}(2 a-1)}{\sqrt{2 a}-1}
$$

which corresponds to $\cos (b Z)=1$. Hence the periodic variation of the coefficients in the NLSE has no effect on the maximum intensity of the KM soliton, which is identical to the one obtained in the case of constant coefficients. On the other hand, the periodic modulation drastically impacts the shape of the KM soliton, that we will analyse through the position, periodic or not, and the local structure of its extrema. Extrema of the KM soliton are critical points of the function $\left|q_{\mathrm{Ma}}\right|_{\max }^{2}$. Hence, following Eq. (7), their positions $z$ satisfy at least one of the two relations:

$$
\begin{gathered}
\sin (b Z)=0, \\
D_{2}(z)=-1+d_{m} \cos \left(k_{m} z\right)=0 .
\end{gathered}
$$

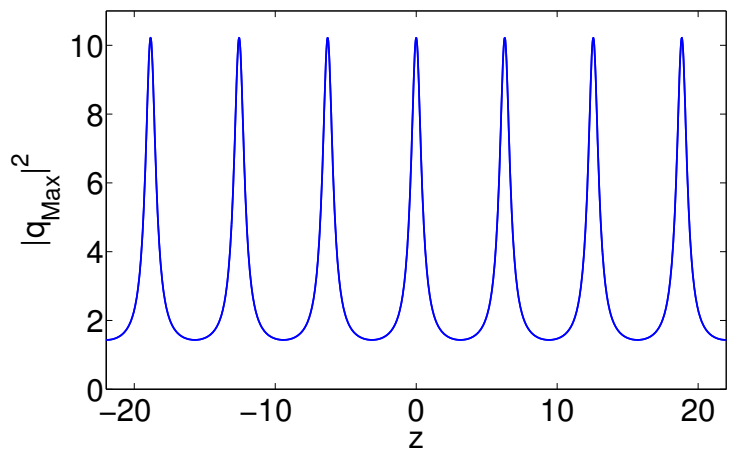

FIG. 2. (Color online) The shape of the Kuznetsov-Ma soliton as a function of $z$ in the case of constant coefficient $d_{m}=0$ with $a=0.603$.
Note that when (9) is satisfied at a given $z$, the function $\left|q_{\mathrm{Ma}}\right|_{\text {max }}^{2}$ reaches either a global maximum or a global minimum depending on the sign of $\cos (b Z)$. When the two equations above are satisfied, simple computations reveal that the five first derivatives of $\left|q_{\mathrm{Ma}}\right|_{\max }^{2}$ vanish. Hence, we refer to such extrema as flat maxima or minima (see Fig 3 and 4 for example). Note the possible presence of other global extrema of $\left|q_{\mathrm{Ma}}\right|_{\max }^{2}$, satisfying only equation (9), but not (10). Those extrema are less flat. Moreover, local extrema satisfy only Eq. (10) (and not $\mathrm{Eq}(9))$ are never flat.

As $d_{m}$ increases from 0 to 1 , condition (10) cannot be satisfied, so that the extrema of the KM soliton are determined by Eq. (9), which is a transcendental equa-

(a)

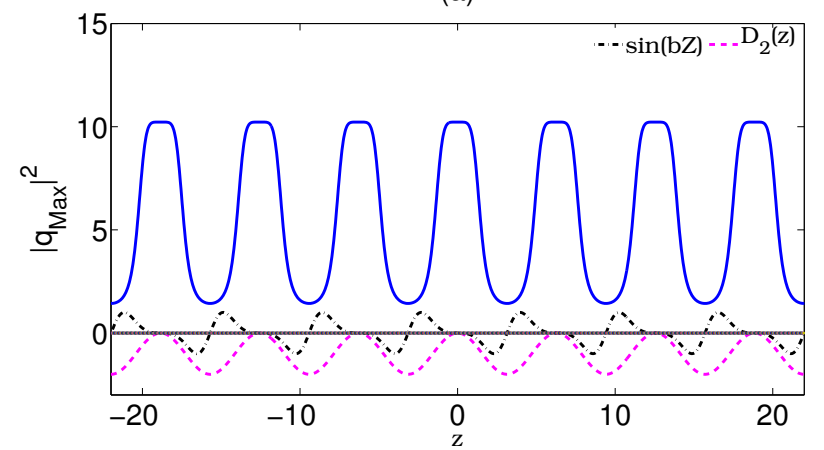

(b)

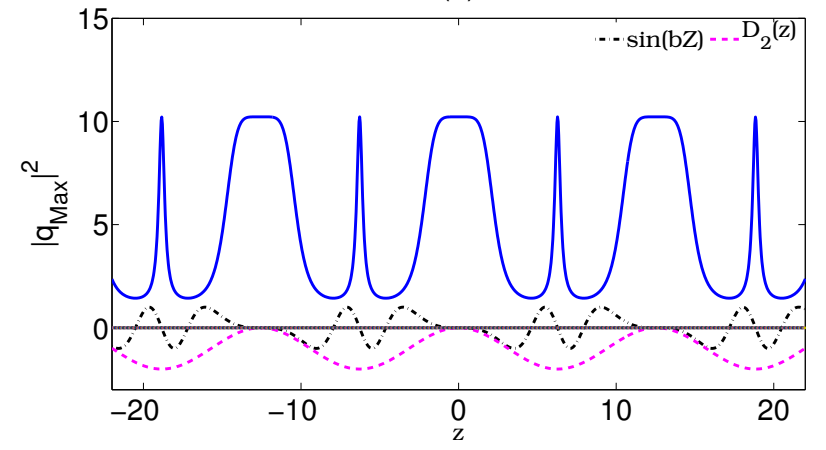

FIG. 3. (Color online) The shape of the generalized Kuznetsov-Ma soliton with periodic coefficients as a function of $z$ with $d_{m}=1$ and $a=0.603$. (a) $k_{m}=b$, (b) $k_{m}=b / 2$. 
tion having no explicit analytical solution. In this regime of the modulation strength $\left(d_{m} \in(0,1)\right)$, the general aspect of the KM soliton remains essentially unaltered, with (quasi-)periodic repetition of its extrema.

For the critical value $d_{m}=1$, Fig. 3 shows a typical example where we see the impact of the frequency of the modulation $k_{m}$ on the shape of the KM soliton. In this case $\left(d_{m}=1\right)$, when $k_{m} / b$ is rational, the two equations (9) and (10) are simultaneously satisfied at points $z_{\ell}$ satisfying

$$
b z_{\ell} \in 2 \pi \frac{N_{2}}{N_{1}} \mathbb{Z} \quad \text { and } \quad b z_{\ell} \in \pi \mathbb{Z} .
$$

In case $N_{1}$ is even, this corresponds exactly to the points

$$
z_{\ell}=\frac{\pi}{b} N_{2} \ell, \quad \ell \in \mathbb{Z},
$$

and they are global maxima if $N_{2} \ell$ is even or global minima if $N_{2} \ell$ odd. In case $N_{1}$ is odd, this corresponds to the points

$$
z_{\ell}=\frac{2 \pi}{b} N_{2} \ell, \quad \ell \in \mathbb{Z},
$$

and all of them are global maxima. In case $d_{m}=1$, any local extremum is global.

(a)

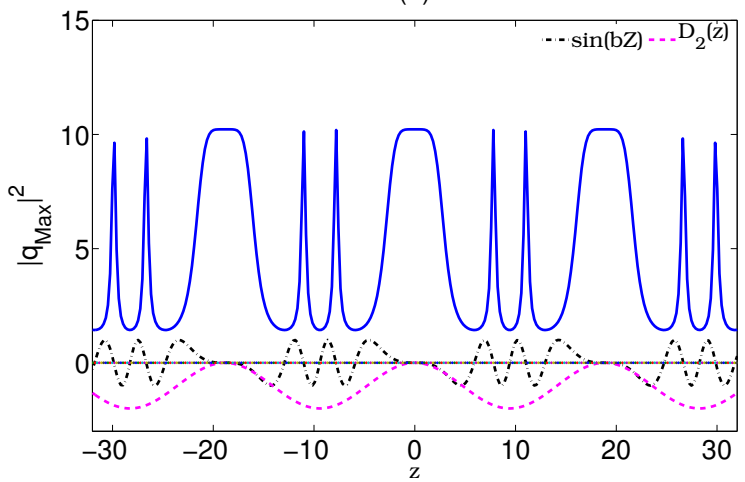

(b)

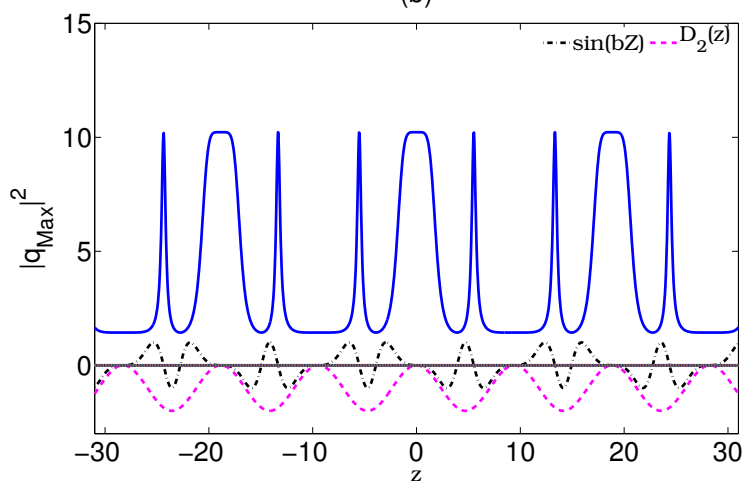

FIG. 4. (Color online) The shape of the generalized Kuznetsov-Ma soliton as a function of $z$ with $a=0.603$ and $d_{m}=1$. (a) $k_{m} / b=1 / 3$, (b) $k_{m} / b=2 / 3$. (a)

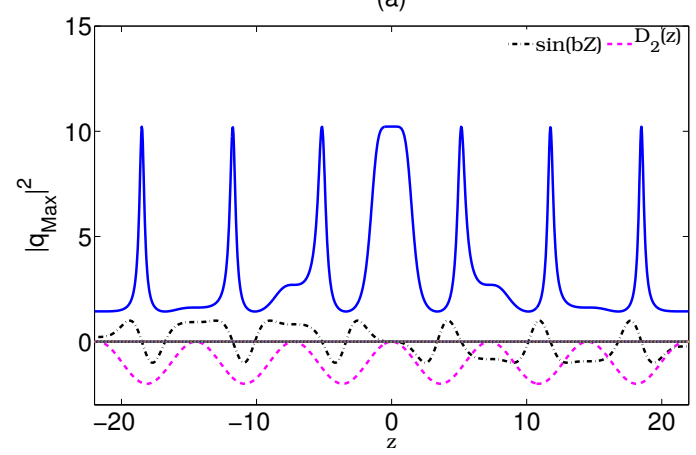

(b)

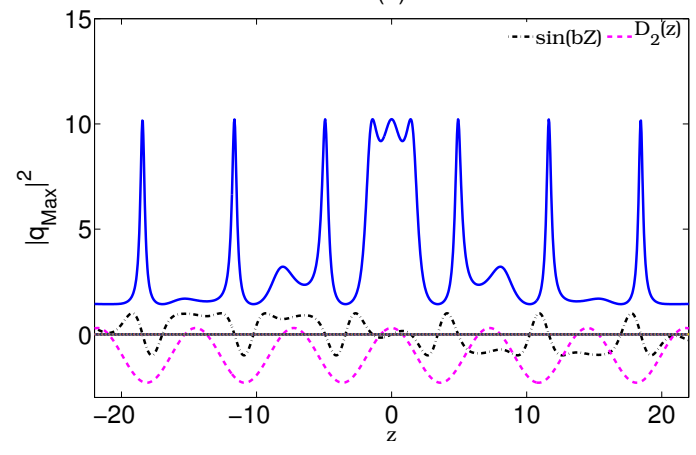

FIG. 5. (Color online) The shape of the generalized Kuznetsov-Ma soliton with periodic coefficients as a function of $z$ with $k_{m}=\sqrt{3} b / 2$ and $a=0.603$. (a) $d_{m}=1$, (b) $d_{m}=1.3$.

Observe that, when $k_{m} / b$ is irrational, there is only one simultaneous solution of Eq. (9) and (10), hence only one flat maximum, centered at $z=0$.

Beyond the critical value $d_{m}=1$, each flat maximum splits in three sharper maxima which are symmetrically located around the central one (see Figs. 6(a), (b)). Note that, when $d_{m}>1$, the function $\left|q_{\mathrm{Ma}}\right|_{\max }^{2}$ also has local maxima and minima. As $d_{m}>1$ increases, the amplitude of local maxima (minima) increases (decreases) to reach the value of the absolute maxima (minima). In case $k_{m} / b$ is rational, this can be seen in Fig. 6. In case $k_{m} / b$ is irrational, this can be observed in Fig. 7 where the position of the arrows shows how the local maxima evolve to the absolute maxima, then widen at the top, before they split in two (Fig. 7(c)).

\section{DIFFERENT REGIMES OF THE OSCILLATIONS OF KUZNETSOV-MA SOLITON IN PRESENCE OF PERIODIC COEFFICIENTS}

In order to shed a different light on the regimes of the spatial oscillations of the KM soliton as a function of the frequency of the modulation, we study the Fourier spectrum and stroboscopic Poincaré sections for the function $\left|q_{\mathrm{Ma}}\right|_{\max }^{2}$ defined in (7) in detail. As we will see, if $k_{m} / b=N_{1} / N_{2}$ is rational, then the Fourier spectrum of $\left|q_{\mathrm{Ma}}\right|_{\max }^{2}$ consists of many subharmonics located on 
a comb of equally spaced frequency components, with $\Delta k=b / N_{2}$. If on the other hand $k_{m} / b$ is irrational, then the frequencies appearing in the spectrum belong to $k_{m} \mathbb{Z}+b \mathbb{Z}$ and are dense in $\mathbb{R}$.

We distinguish three cases : first $k_{m} / b=1$, then $k_{m} / b \neq 1$ rational and finally $k_{m} / b$ irrational. For $k_{m}=b,\left|q_{\mathrm{Ma}}\right|_{\max }^{2}$ is periodic and has the same period as the external modulation of the dispersion $D_{2}$. In that case, the discrete phase-space trajectory obtained by evaluating $\left(\left|q_{\mathrm{Ma}}\right|_{\max }^{2},\left(\left|q_{\mathrm{Ma}}\right|_{\max }^{2}\right)_{z}\right)$ at each period (stroboscopic Poincaré section) $2 \pi / k_{m}$ of the modulation, starting from $z=0$, obviously reduces to a single point (see

(a)

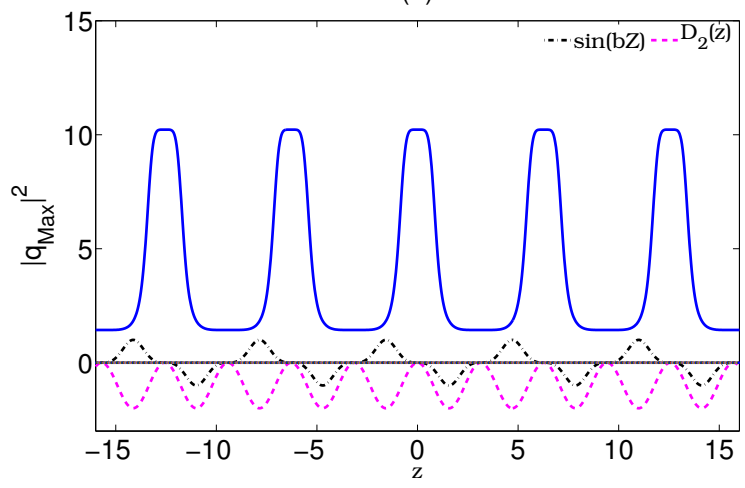

(b)

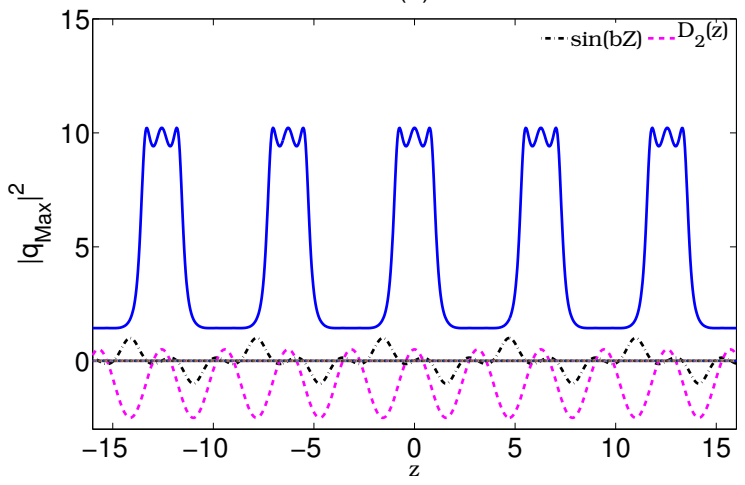

(c)

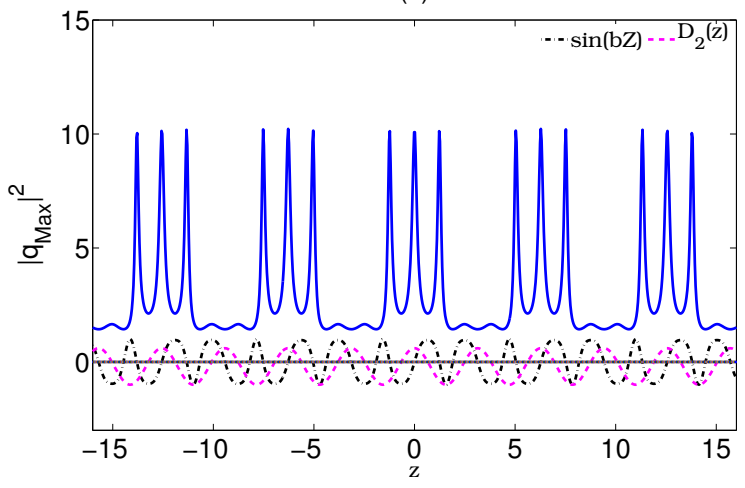

FIG. 6. (Color online) The shape of the generalized Kuznetsov-Ma soliton with periodic coefficients as a function of $z$ with $k_{m}=2 b$ and $a=0.603$. (a) $d_{m}=1$, (b) $d_{m}=1.5$, (c) $d_{m}=4.0$
Fig. 8(a)) and the Fourier spectrum contains a discrete set of equally distant frequency components at integer multiples of $k_{m}$.

More generally, when the ratio $k_{m} / b=N_{1} / N_{2}$ with $N_{1}$ and $N_{2}$ mutually prime integer numbers, the signal $\left|q_{\mathrm{Ma}}\right|_{\max }^{2}$ is still periodic (with period $z_{*}=2 \pi N_{1} / k_{m}$ ), but displays sharp pulses (see Fig. 8(b)), due to specific relations between its Fourier components, reminiscent of phase-locking [33, 34]. The phase space diagram is consequently composed of a set of $N_{1}$ points. This can be seen in the middle panel of Fig. 8(b) for $k_{m} / b=5 / 2$ where we have numbered $P_{j}(j=1, \ldots, 5)$, the successive points in the Poincaré section. In this case, everything happens as if a nonlinear resonance occurred between the frequency $k_{m}$ of the modulation and the intrinsic frequency $b$ of the KM soliton.

Finally, as already pointed out in Sec. III, when $k_{m} / b$ is an irrational number, the function $\left|q_{\mathrm{Ma}}\right|_{\max }^{2}$ is quasiperiodic. In this case, the frequency $k_{m}$ of the modulation and the natural frequency $b$ of the KM soliton are incommensurate and as a result, the oscillations are more complex, but of course not chaotic. The obtained stroboscopic phase-space trajectory never returns to the same point. Instead, it traces out a closed loop (see Fig $8(\mathrm{c}))$. This can be explained as follows. Introducing the $2 \pi$-periodic function

$$
G\left(\theta_{1}, \theta_{2}\right)=1+\frac{4 \sqrt{2 a}(2 a-1)}{\sqrt{2 a}-\cos \left(-\theta_{1}+b \frac{d_{m}}{k_{m}} \sin \left(\theta_{2}\right)\right)},
$$

in two angular variables $\theta_{1}, \theta_{2}$ and setting $\nabla G=$ $\left(\partial_{\theta_{1}} G, \partial_{\theta_{2}} G\right)$, one notes that the phase-space trajectory $z \mapsto\left(\left|q_{\mathrm{Ma}}\right|_{\max }^{2}(z),\left(\left|q_{\mathrm{Ma}}\right|_{\max }^{2}\right)_{z}(z)\right)$ is the image of the trajectory on the torus

$\theta(z)=\left(\theta_{1}(z), \theta_{2}(z)\right), \quad$ with $\theta_{1}(z)=b z$ and $\theta_{2}(z)=k_{m} z$,

by the function $\left(G,\left(b, k_{m}\right) \cdot \nabla G\right)$. Indeed, by Eq. (7), we have

$$
\left|q_{\mathrm{Ma}}\right|_{\max }^{2}(z)=G(\theta(z))
$$

and

$$
\left(\left|q_{\mathrm{Ma}}\right|_{\max }^{2}\right)_{z}(z)=\theta^{\prime}(z) \cdot((\nabla G)(\theta(z))) .
$$

Recall that the points $\left(P_{j}\right)_{j \in \mathbb{N}}$ correspond to values of $z$ of the form $z_{j}=j 2 \pi / k_{m}+z_{0}$, so that $\theta_{2}\left(z_{j}\right)=\theta_{2}\left(z_{0}\right)[2 \pi]$, independently of $j$, and consequently the stroboscopic phase-space points fill the one-dimensional loop $\theta_{1} \mapsto$ $\left(G\left(\theta_{1}, \theta_{2}\left(z_{0}\right)\right),\left(b, k_{m}\right) \cdot \nabla G\left(\theta_{1}, \theta_{2}\left(z_{0}\right)\right)\right)$. Many subharmonics appear again in the Fourier spectrum, but they are not equally spaced as before. Instead, additional frequencies occur at all values of the form $n_{1} b+n_{2} k_{m}$ with $n_{1}$ and $n_{2}$ arbitrary integers. This is illustrated in Fig. 8 (c) for $k_{m} / b=\sqrt{3} / 2$. It is to be noted that the side frequencies that significantly appear in the spectrum (whether $k_{m} / b$ is rational or not) are determined by the strength $d_{m}$ of the modulation as we will illustrate below. 
(a)

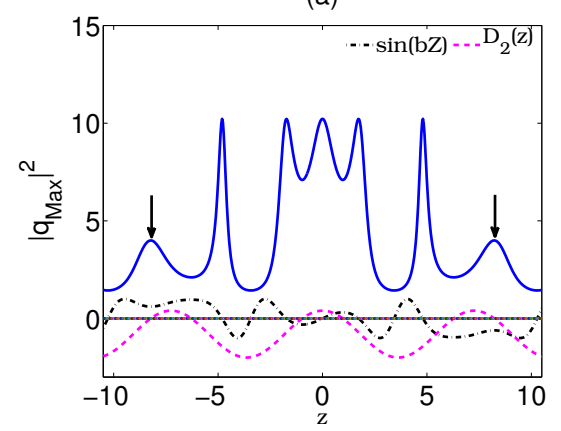

(b)

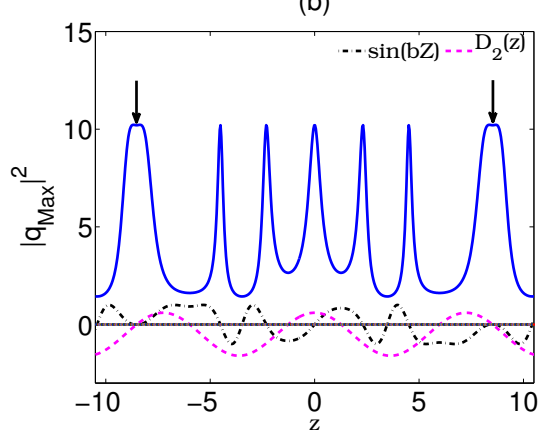

(c)

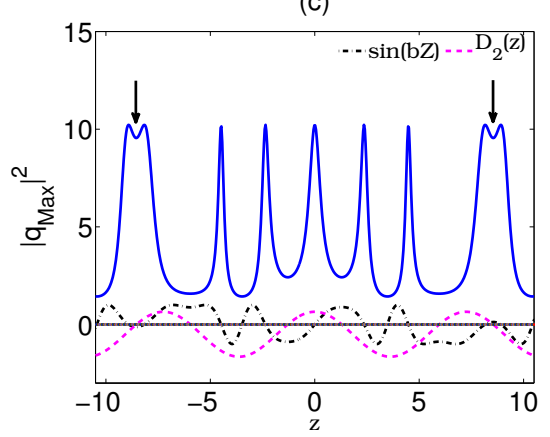

FIG. 7. (Color online) The shape of the generalized Kuznetsov-Ma soliton as a function of $z$. (a) $d_{m}=1.5$, (b) $d_{m}=2.2$, (c) $d_{m}=2.3$. The other parameters are $k_{m}=\sqrt{3} b / 2, a=0.603$.

(a)
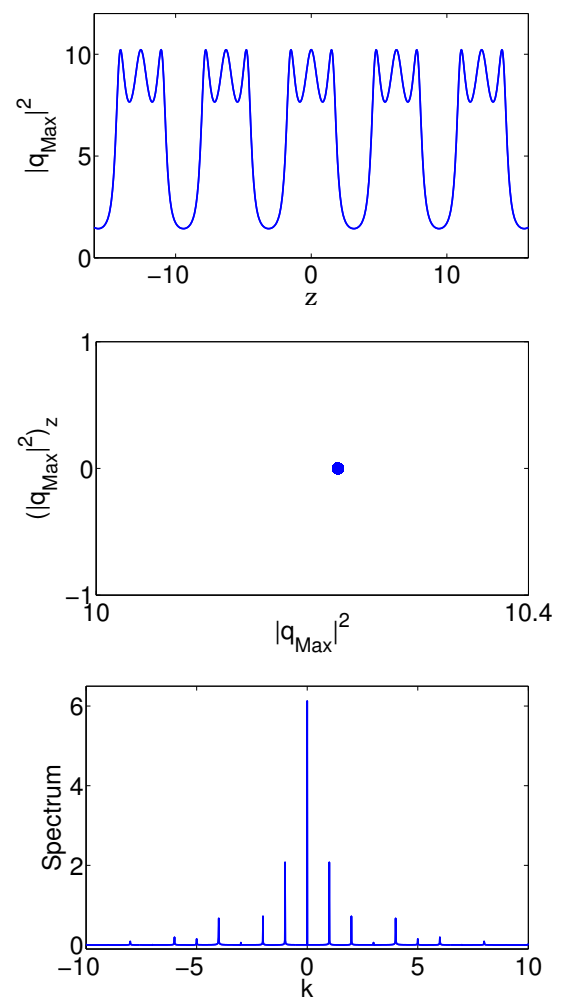

(b)
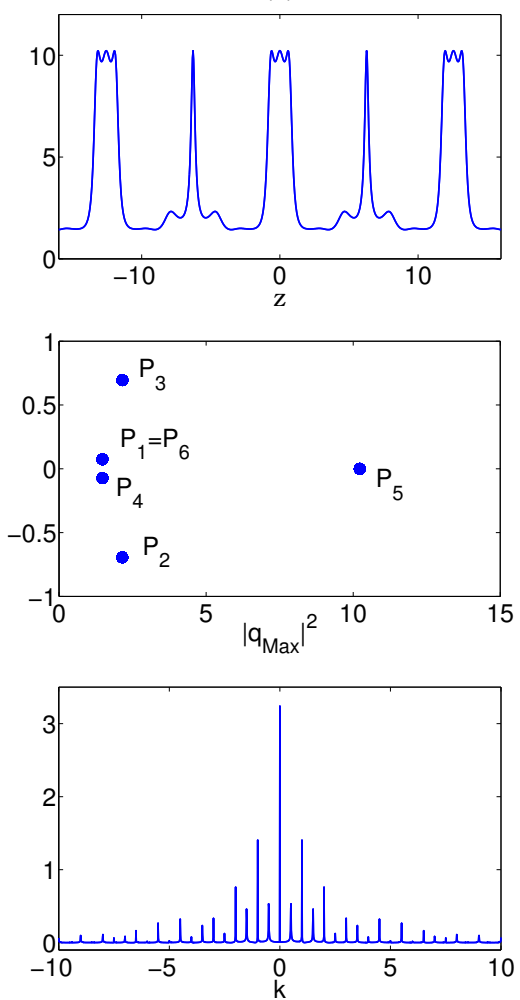

(c)
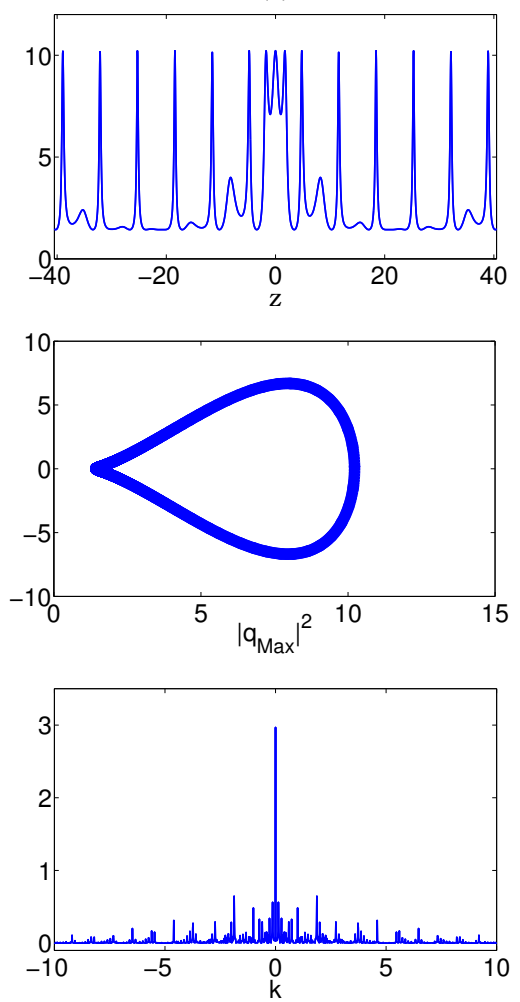

FIG. 8. (Color online) Evolution of the maximum intensity of the Kuznetsov-Ma soliton as a function of $z$ (upper panels); phase space diagram (middle panels); evolution of the Fourier spectrum (lower panels). (a) $k_{m}=b,(\mathrm{~b}) k_{m}=5 b / 2,(\mathrm{c}) k_{m}=\sqrt{3} b / 2$. The other parameters are $a=0.603, d_{m}=1.5$.

As $d_{m}$ increases, the energy content that is initially concentrated, when $d_{m}=0$, in the carrier frequency $b$ and its harmonics is distributed among an increasing number of sidebands. However the relation

$$
\int_{-\infty}^{+\infty}\left(\left|q_{M a}(t, z)\right|^{2}-1\right) d t=2 \omega
$$

implies that the total energy is conserved, as expected. Similar results have been obtained in [35] where extreme wave phenomena in down-stream running modu- lated waves have been investigated.

To better explain the appearance of additional sideband frequencies as $d_{m}$ is increased, we consider the asymptotic case where $a \gg 1$. In this limit, Eq. can be approximated by:

$$
\left|q_{\mathrm{Ma}}\right|_{\max }^{2}(z)=8 a+4 \sqrt{2 a} \cos (b Z)
$$

Now, the cosine function can readily be decomposed into 
its component frequencies as follows

$$
\begin{array}{r}
\cos \left[b z-\beta \sin \left(k_{m} z\right)\right]=J_{0}(\beta) \cos (b z) \\
+J_{1}(\beta)\left[\cos \left(b-k_{m}\right) z-\cos \left(b+k_{m}\right) z\right] \\
+J_{2}(\beta)\left[\cos \left(b-2 k_{m}\right) z+\cos \left(b+2 k_{m}\right) z\right] \\
+J_{3}(\beta)\left[\cos \left(b-3 k_{m}\right) z-\cos \left(b+3 k_{m}\right) z\right] \\
\ldots \\
+J_{n}(\beta)\left[\cos \left(b-n k_{m}\right) z+(-1)^{n} \cos \left(b+n k_{m}\right) z\right] \\
+\ldots,
\end{array}
$$

where $b \approx 4 a$, and where $\beta=d_{m} b / k_{m}$ is the modulation index.

It can be seen from the above relation that the amplitudes of the carrier and sideband components in the spectrum of the KM soliton are determined by the $n t h$ order Bessel functions of the first kind $J_{n}(\beta)$. For small values of the modulation index, $J_{0}(\beta) \simeq 1$ and $J_{n}(\beta) \simeq \beta^{n}$ and the spectrum of the KM soliton is very close to the one obtained in absence of modulation. This can be seen in the spectrum of Fig. 9(a) corresponding to $\beta=0.1$ where the peak amplitude of the carrier frequency $k=b$ is clearly strongly dominant. Further increase of $\beta$ results in successive side frequencies and the relative amplitudes of all the frequencies present in each specific case depend on the values of the Bessel functions involved. Some of the amplitudes, that of the carrier included, can be suppressed completely when the index $\beta$ coincides with a root of the corresponding Bessel function. For example, $d_{m}=0.6$ and $k_{m}=b / 4$ correspond to $\beta=2.4$. The amplitude of the carrier frequency which is controlled in this case by $J_{0}(2.4)$ and $J_{8}(2.4)$ vanishes in the spectrum as shown in Fig. 9(b). This is a striking and an important issue since it means that external modulation $\left(d_{m}\right.$ and $k_{m}$ ) can be tuned to shift at will the spectral frequencies of the system.

\section{CONCLUSION}

We have carried out a theoretical investigation of the generalized Kuznetsov-Ma soliton of a completely integrable nonlinear Schrödinger equation with periodically varying coefficients. The impact of the periodic modulation on the shape of the generalized Kuznetsov-Ma soliton has been analyzed. The amplitude of the modulation has no effect on the maximum intensity of the KuznetsovMa soliton, but it promotes the development of multiple compression points. On the other hand, the frequency of the modulation drastically impacts the shape of the solution. We have then investigated the nonlinear nature of the Kuznetsov-Ma soliton's intensity as a function of the frequency of the modulation. The regime of the oscillations were studied in the light of the dynamical systems tools such as Poincaré sections and Fourier spectrum. It was found that when the ratio between the natural frequency of the Kuznetsov-Ma soliton and the frequency of the modulation is rational, a nonlinear
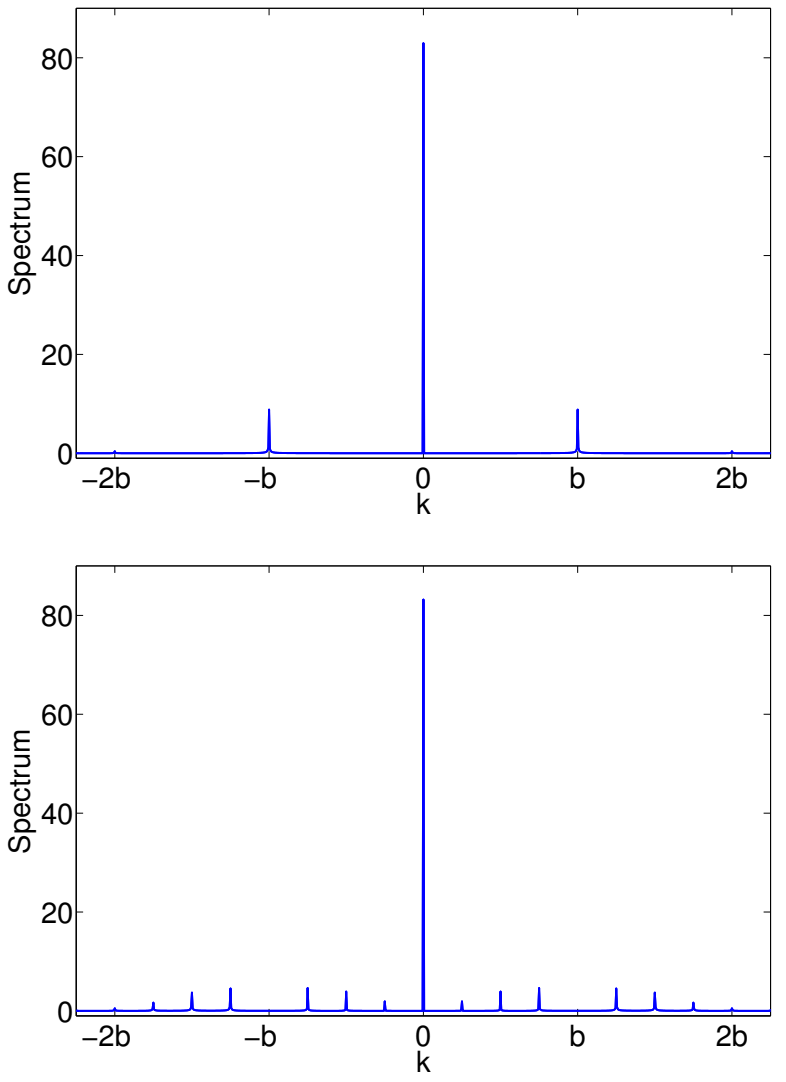

FIG. 9. (Color online) The spectrum of the KM soliton with periodic modulation for $a=10.255$. (a) $d_{m}=0.1, k_{m}=b$, (b) $d_{m}=0.6, k_{m}=\frac{b}{4}$.

resonance occurs between the two frequencies and the Kuznetsov-Ma soliton's intensity is a periodic function. In the case where the ratio of the above two frequencies are irrational, quasiperiodic oscillations were observed. A striking and important result from our investigation is the possibility to suppress either the carrier frequency or any subharmonic by a judicious choice of the amplitude and frequency of the modulation. One consequence is that external modulation can be tuned to experimentally shift at will the spectral frequencies of the system. This makes it possible to control the formation and the properties of rogue waves of the Kuznetsov-Ma type. The extension of these theoretical results to a large class of nonintegrable systems with modulated coefficients will be the subject of further investigation.

\section{ACKNOWLEDGEMENTS}

C. G. L. T. acknowledges the support of the "Laboratoire d'Excellence CEMPI: Centre Européen pour les Mathématiques, la Physique et leurs Interactions" (ANR11-LABX-0007-01). This research was supported in part by the Interuniversity Attraction Poles program of the 
Belgium Science Policy Office under the grant IAPP7-35 and the French Agence Nationale de la Recherche project OptiRoc ANR-12-BS04-0011.
[1] L. Drape, Mar. Obs. 35, 193 (1965); K. Dysthe, H. E. Krogstad, P. Muller, Annu. Rev. Fluid Mech. 40, 287 (2008); C. Kharif, E. Pelinovsky, and A. Slunyaev, Rogue waves in the ocean, (Springer-Verlag, 2009).

[2] B. Kibler, J. Fatome, C. Finot, G. Millot, F. Dias, G. Genty, N. Akhmediev, and J. M. Dudley, Nature Phys. 6, 790 (2010).

[3] D. R. Solli, C. Ropers, P. Koonath, B. Jalali, Nature 450, 1054 (2007).

[4] M. Shats, H. Punzmann, and H. Xia, Phys. Rev. Lett. 104, 104503 (2010).

[5] Y. V. Bludov, V. V. Konotop, and N. Akhmediev, Phys. Rev. A 80 033610, (2009).

[6] W. M. Moslem, P. K. Shukla, and B. Eliasson, Eur. Phys. Lett 96, 25002, (2011).

[7] L. Stenflo and M. Marklund, J. Plasma Phys. 76 293, (2010).

[8] N. Akhmediev, A. Ankiewicz, and M. Taki, Phys. Lett. A 373, 675 (2009).

[9] N. Akhmediev, J. M. Dudley, D. R. Solli, and S. K. Turitsyn, J. Opt. 15, 060201 (2013).

[10] N. Akhmediev, J. M. Soto-Crespo, and A. Ankiewicz, Phys. Lett. A, 373, 2137 (2009).

[11] J. M. Dudley, F. Dias, M. Erkintalo, and G. Genty, Nature Photonics 8, 755 (2014).

[12] S. Toenger, T. Godin, C. Billet, F. Dias, M. Erkintalo, G. Genty, and J. M. Dudley, Sci. Rep. 5, 10380 (2015).

[13] M. Onorato, S. Residori, U. Bortolozzo, A. Montina, and F. T. Arecchi, Phys. Reports 528, 47 (2013).

[14] E. A. Kuznetsov, Sov. Phys. Dokl. 22, 507 (1977).

[15] Y. C. Ma, Stud. Appl. Math. 60, 43 (1979).

[16] N. Akhmediev and V. I. Korneev, Theor. Math. Phys. 69, 1089 (1986).

[17] D. H. Peregrine, J. Aust. Math. Soc. 25, 16 (1983).

[18] A. Mussot, A. Kudlinski, M. Kolobov, E. Louvergneaux, M. Douay, and M. Taki, Opt. Express 17, 17010 (2009).

[19] K. Hammani, C. Finot, J. M. Dudley, and G. Millot, Opt. Express 16, 16467 (2008); G. Yang, Y. Wang, Z. Qin, B. A. Malomed, D. Mihalache, and L. Li, Phys. Rev. E 90, 062909 (2014).

[20] B. Frisquet, B. Kibler, and G. Millot, Phys. Rev. X 3, 041032 (2013).

[21] B. Kibler, J. Fatome, C. Finot, G. Millot, G. Genty, B. Wetzel, N. Akhmediev, F. Dias, and J. M. Dudley, Sci.
Rep. 2, 463 (2012).

[22] L. Wang, X. Li, F. H. Qi, and L. L. Zhang, Ann. Phys. 359, 97 (2015); L. Wang, M. Li, F. H. Qi, and X. Tao, phys. plasmas 22, 032308 (2015); L. Wang, M. Li, F. H. Qi, and C. Geng, Eur. Phys. J. D 69, 108 (2015).

[23] A. Bendahmane, A. Mussot, P. Szriftgiser, O. Zerkak, G. Genty, J. M. Dudley, and A. Kudlinski, Opt. Lett. 39, 4490 (2014).

[24] C. G. L. Tiofack, S. Coulibaly, M. Taki, S. De Bièvre, and G. Dujardin, Phys. Rev. A 92, 043837 (2015).

[25] Q. Tian, Q. Yang, C. Q. Dai, and J. F. Zhang, Opt. Commun. 284, 2222(2011); C. Q. Dai, C. L. Zheng, and H. P. Zhu, Eur. Phys. J. D 66, 112 (2012); C. N. Kumar, R. Gupta, A. Goyal, S. Loomba, T. S. Raju, and P. K. Panigrahi, Phys. Rev. A 86025802 (2012).

[26] H. P. Zhu, Z. H. Pan, and J. P. Fang, Eur. Phys. J. D 68, 69 (2014); C. Q. Dai, Y. Wang, and X. Zhang, Opt. Express 22, 29862 (2014); C. Q. Dai and W. H. Huang, Phys. Lett. A 378, 1113 (2014).

[27] U. Al. Khawaja, S. M. Al-Marzoug, H. Bahlouli, and F. Kh. Abdullaev, Commun Nonlinear Sci Numer Simulat. 32, 1 (2016).

[28] G. P. Agrawal, Nonlinear Fiber Optics, 3rd ed. (Academic, Boston, 2008).

[29] V. N. Serkin and A. Hasegawa, Phys. Rev. Lett. 85, 4502 (2000).

[30] V. N. Serkin and A. Hasegawa, JETP Lett. 72, 89 (2000); V. N. Serkin and A. Hasegawa, IEEE J. Sel. Top. Quant. Electron. 8, 418 (2002).

[31] R. Hao, L. Li, Z. Li, W. Xue, and G. Zhou, Opt. Commun. 236, 79 (2004).

[32] Y. Wang, L. Song, L. Li, and B. A. Malomed, J. Opt. Soc. Am. B 32, 2257 (2015).

[33] P. Berge, Y. Pomeau, and Ch. Vidal, L'ordre dans le chaos, (Hermann, 1984).

[34] K. H. Spatschek, M. Taki, and TH. Eickermann, Nonlinear Coherent Structures, Lectures Notes in Physics, 353, (Springer-Verlag, 1990).

[35] Andonowati, N. Karjanto, and E. van Groesen, App. Math. Mode 31, 1425 (2007). 